\title{
Análisis fisicoquímico del aceite crudo de palma proveniente del hibrido interespecífico fortuna en una plantación ubicada en Cabuyaro-Meta
}

Physicochemical analysis of crude palm oil from the interspecific fortune

hybrid in a plantation located in Cabuyaro-Meta

\section{Análise físico-química do óleo de palma bruto do híbrido interespecífico da}

\section{fortuna em uma plantação localizada em Cabuyaro-Meta}

\author{
Acuña Herrera Cristian Fabián ${ }^{1}$, Quesada Ramírez Rafael Andrés ${ }^{1}$ y \\ Lopez Muñoz Luis Gilberto \\ ${ }^{1}$ Ingenieros Agroindustriales, Universidad de los Llanos y \\ ${ }^{2}$ Ingeniero Agroindustrial, Docente Universidad de los Llanos \\ llopezm@unillanos.edu.co
}

Recibido 03 de Diciembre 2018, Aceptado 27 de Mayo 2019

\section{RESUMEN}

La agroindustria de la palma de aceite es de las más promisorias del país. Hoy en día para posicionar un código en el mercado de las semillas, es importante contar con la ficha técnica del producto a comercializar. Las investigaciones adelantadas por Hacienda La Cabaña dieron como resultado un nuevo hibrido denominado Fortuna, que presento mayor rendimiento en fruta fresca y mayor porcentaje de extracción de aceite. El objetivo de este trabajo, fue analizar fisicoquímicamente el aceite de palma crudo, del híbrido Interespecífico Elaeis oleifera Coari x Elaeis guineensis La Mé, denominado Fortuna, para poder comparar los resultados con los valores teóricos del aceite del híbrido Interespecífico Classic e identificar los parámetros de calidad del aceite crudo de palma de acuerdo a la NTC 431. Los resultados obtenidos con la palma tradicional y el híbrido Classic muestran que algunos componentes de ácidos grasos conservan sus valores relativos muy similares, sin embargo, el aceite del hibrido código Fortuna tiene más triglicéridos que contienen ácido oleico, y un menor contenido de ácido palmítico. 
Palabras clave: Palma aceitera, oleaginosas, aceite vegetal, ácidos grasos, ácido oleico.

\section{ABSTRACT}

Agribusiness palm oil is the most promising country. Today for positioning a code in the seed market, it is important to have the data sheet for the product to market. The investigations conducted by Hacienda La Cabaña resulted in a new hybrid called Fortuna, which had higher fresh fruit yield and higher percentage of oil extraction. The aim of this study was to analyze physicochemically crude palm oil, interspecific hybrid Elaeis oleifera x Elaeis guineensis Coari La Mé, called Fortuna, to compare the results with theoretical values interspecific hybrid oil Classic and identify quality parameters of crude palm oil according to NTC 431. The results obtained with the traditional palm and the classic hybrid show that some fatty acid components retain their very similar relative values, however oil hybrid Fortuna code has more triglycerides containing oleic acid, and lower in palmitic acid.

Keywords: Oil palm, oilseeds, vegetable oil, fatty acids, oleic acid.

\section{RESUMO}

O agronegócio do dendê é um dos mais promissores do país. Hoje em dia para posicionar um código no mercado de sementes, é importante ter a ficha técnica do produto a ser comercializado. As investigações realizadas pela Hacienda La Cabaña resultaram em um novo híbrido chamado Fortuna, que apresentou maior rendimento em frutas frescas e maior porcentagem de extração de óleo. O objetivo deste trabalho foi analisar fisicoquimicamente o óleo de palma cru do híbrido Interspecífico Elaeis oleifera Coari x Elaeis guineensis La Mé, denominado Fortuna, a fim de comparar os resultados com os valores teóricos do óleo do híbrido Interspecific Classic e identificar Parâmetros de qualidade do óleo de palma bruto de acordo com a NTC 431. Os resultados obtidos com a palma tradicional e o híbrido Classic mostram que alguns componentes de ácidos graxos retêm seus valores relativos muito semelhantes, no entanto, o óleo híbrido Fortuna tem mais triglicerídeos contendo ácido oleico e um menor teor de ácido palmítico. 
Palavras-chave: Óleo de palma, oleaginosas, óleo vegetal, ácidos gordos, ácido oleico.

\section{INTRODUCCIÓN}

La palma aceitera pertenece a la familia Palmaceae, tribu Cocoineae. Jacquin, la describió en 1763 y le dio el nombre de Elaeis guineensis; este género incluye tres especies: E. guineensis, de África Occidental; E. oleífera (Elaeis melanococa), que se extiende de Centroamérica a Brasil, y E. odora, una especie poco conocida de América del Sur (Ortiz, 1994). Es una planta perenne, cultivada por su alta productividad de aceite, la especie tiene tres variedades: dura, tenera y pisifera; de ellas la variedad tenera es la que se utiliza comercialmente para la extracción del aceite y es un cruce entre las otras dos variedades (Quesada, s.f).

La palma de aceite es una planta del trópico propia de climas cálidos y es la más productiva de las oleaginosas del planeta, en una hectárea presenta un rendimiento de aceite entre seis y diez veces superior al de otros cultivos, como la soya y el girasol entre otros (Cenipalma, 2006). Tiene en promedio una vida que oscila entre los 24 y los 28 años, de acuerdo con el tipo de material plantado, expresa mejor su potencial de producción en condiciones de alta temperatura, buena radiación solar, alta precipitación y humedad relativa (Brugés,2004). Alrededor de 8,6 millones de hectáreas del mundo sembradas con palma de aceite abastecen el $23 \%$ de la demanda mundial de aceites y grasas, mientras que 88 millones de hectáreas de soya sólo abastecen el $24 \%$ de ella (Cenipalma, 2013).

El aceite de palma se viene consumiendo desde hace más de 5000 años (Quesada, s.f.), y es uno de los productos de mayor crecimiento en la industria colombiana. El aceite se extrae del mesocarpio del fruto de la semilla de la palma africana Elaeis guineensis Jacq., a través de procedimientos mecánicos; está constituido por una mezcla de ésteres de glicerol (triglicéridos) y es fuente natural de carotenos y vitamina E, gracias a su versatilidad, dada por su composición de ácidos grasos saturados e insaturados (Rincón y Martínez, 2009). 
El aceite de palma contiene iguales proporciones de ácidos grasos no saturados, conteniendo alrededor del $40 \%$ de ácido oleico (no monosaturado), $10 \%$ de ácido linoléico (no polisaturado), 44\% de ácido palmítico (saturado) y 5\% de ácido esteárico (saturado) (Quesada, s.f.). Es la fuente natural más rica en vitamina $E$, antioxidante que protege el cerebro y el corazón, protege contra el cáncer y enfermedades crónicas como el Alzheimer, es naturalmente libre de grasas trans y protege la salud cardiovascular (Fedepalma, 2016). Los carotenos, se hayan presentes en concentraciones del orden de partes por millón (ppm), son sustancias de gran importancia para la estabilidad del aceite de palma africana porque previenen la oxidación causada por el oxígeno del aire y que además es acelerada por las altas temperaturas y los rayos UV de la luz solar. El aceite crudo de palma también contiene cierta cantidad de agua que puede ser generadora por la hidrólisis de los triglicéridos si se exceden niveles críticos, esta humedad proviene en parte de los mismos frutos de la palma, o del agua añadida en las diferentes etapas del proceso de obtención del aceite (Fontalvo et al., 2014).

El sector palmero continúa posicionándose como una de las alternativas de desarrollo más promisorias del agro colombiano, a nivel mundial Colombia se posiciona como el cuarto productor de aceite de palma y el primero en América Latina (Fedepalma, 2016). En el país, la palma de aceite se identifica por zonas, en la oriental, se identificaron 29 núcleos palmeros, en esta zona, los productores de palma del Meta y Casanare ocupan el primer lugar a nivel nacional en cuanto al área sembrada y la producción de toneladas de aceite; actualmente el departamento del Meta cuenta con 20 municipios con áreas sembradas en palma de aceite (Fedepalma, 2016), entre los cuales se encuentra Hacienda La Cabaña S.A., ubicada en el municipio de Cabuyaro Meta.

Hacienda La Cabaña dentro sus investigaciones ha caracterizado en producción un nuevo código de palma híbrido (OxG) en el cual se evaluaron ocho pólenes de $E$. guineensis con 23 madres $E$. oleífera, buscando optimizar el rendimiento en campo, y variedades que sean resistentes a plagas y enfermedades; de estas investigaciones surgió el híbrido denominado FORTUNA, que por su mayor 
rendimiento en fruta fresca y mayor porcentaje de extracción dando como resultado 7.2 toneladas de aceite al año por hectárea, se convierte en un nuevo código de palma prometedor en el sector palmero en un futuro no muy lejano.

En este orden de ideas, Hacienda La Cabaña busca posicionar este código en el mercado de semillas, para ello es importante tener una ficha técnica del producto a comercializar, por lo que se debe caracterizar las propiedades del fruto para realizar las respectivas comparaciones con la variedad que se trabaja actualmente de palma comercial (E. guineensis Híbrido Interespecífico Classic). Por lo tanto, en este trabajo se analizó fisicoquímicamente el aceite de palma crudo del híbrido denominado FORTUNA comparando los resultados obtenidos con los valores teóricos del híbrido Interespecífico Classic, siendo éste el de mayor comercialización, y finalmente identificar los parámetros de calidad del aceite crudo de palma de acuerdo a la Norma Técnica Colombiana para aceite crudo natural de palma africana NTC 431 (ICNTC, 2009).

\section{METOdOLOGÍA}

Este estudio se realizó en los laboratorios de la Universidad de los Llanos, en la empresa Hacienda La Cabaña S.A., y el Centro de Investigaciones en Palma de Aceite (CENIPALMA), para lo cual se analizaron cinco (5) racimos maduros de hibrido código Fortuna obtenidos de palmas diferentes, en cinco (5) repeticiones. Los frutos de palma fueron recolectados en un área de 20 hectáreas de un cultivo de 15 años de establecido, en el cual se encuentran distribuidos diferentes híbridos y/o variedades a los que se les asignó un código determinado, lo cuales hacen parte de los ensayos de la empresa Hacienda La Cabaña; el código fortuna está distribuido en sitios diferentes con 12 palmas cada uno, de los cuales se seleccionaron cinco sitios en los que se caracterizó la palma y se muestreó un racimo de cada una, el área de estudio estuvo definida con un consecutivo de línea palma número 21-128.

En la primera parte del estudio se obtuvieron todas las muestras de racimos recién cosechados (Figura 1), los cuales se trasladaron cuidadosamente desde el campo 
hasta el laboratorio donde se pesaron en una balanza eléctrica (Figura 2), donde posteriormente se escogieron los frutos que no mostraron ningún tipo de alteración física.
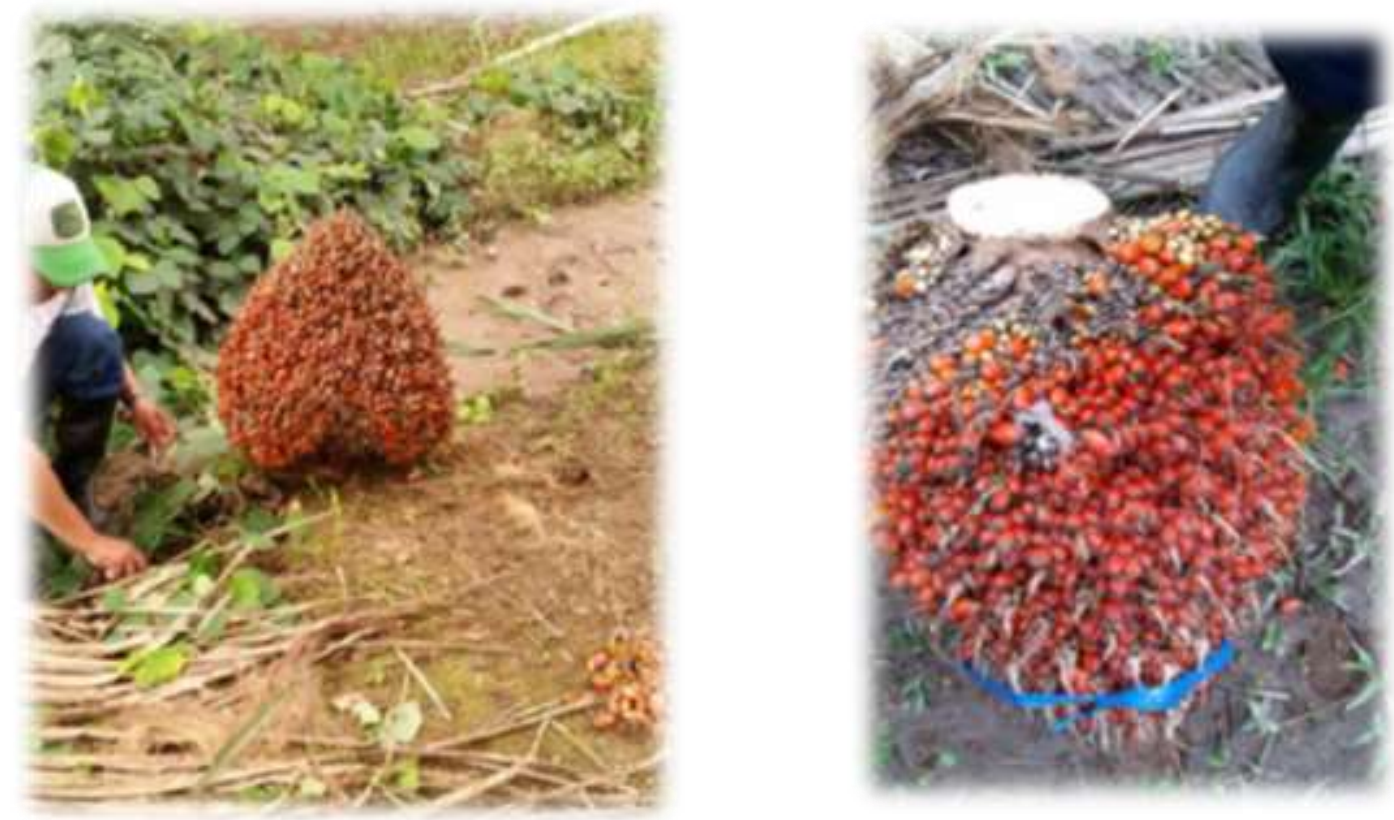

Figura 1. Recolección en campo del racimo de palma código Fortuna
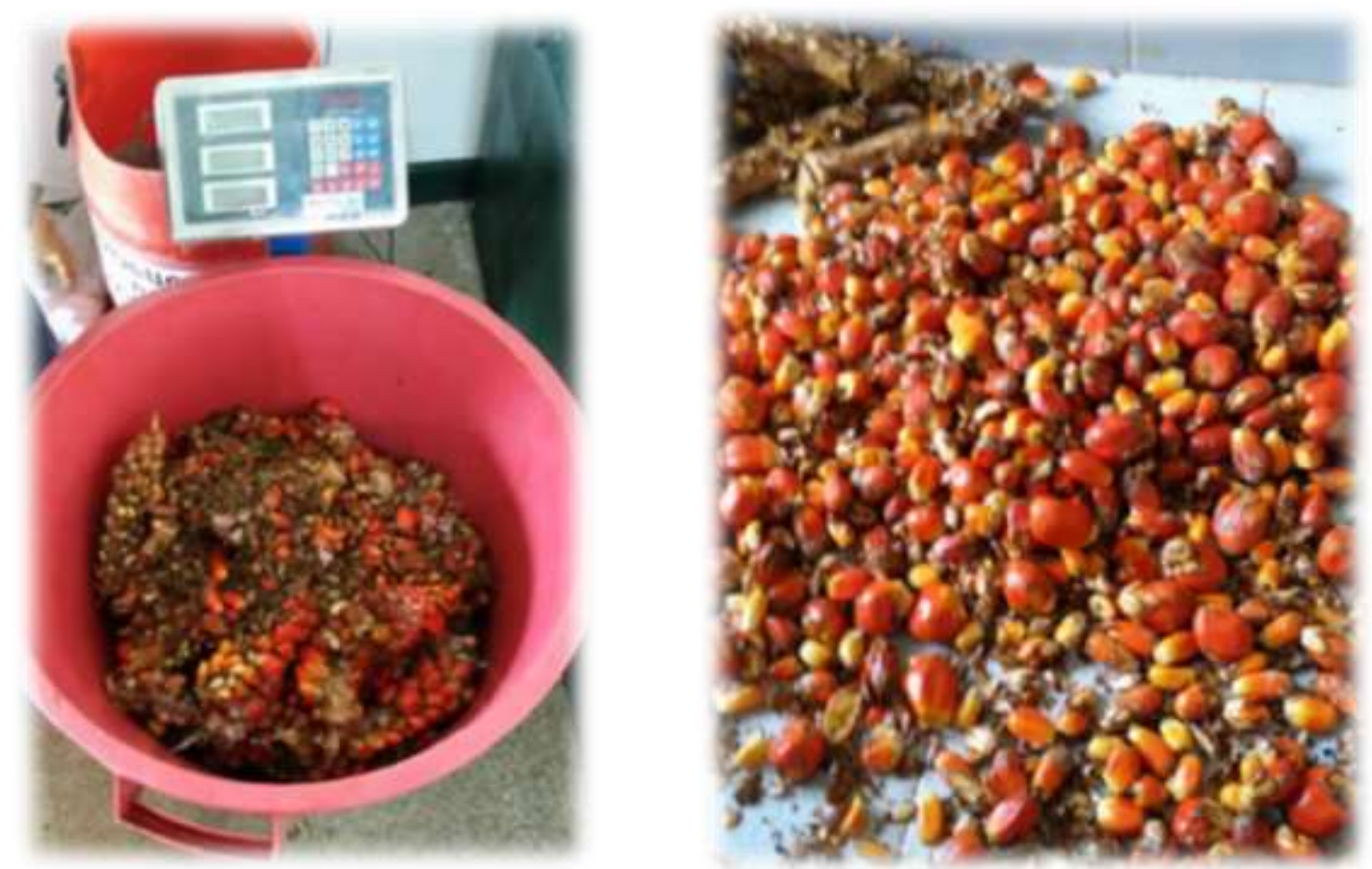

Figura 2. Pesaje del racimo de palma código Fortuna 
Posteriormente se separaron los racimos (Figura 3) en lonas, luego se colocaron al interior de recipientes cilíndricos horizontales (autoclave), donde los racimos se cocinaron con el vapor generado por una caldera durante 45 minutos. Una vez esterilizados los racimos, se realizó el desfrutamiento, el cual consiste en separar de los racimos esterilizados los frutos sueltos. Finalmente, los frutos se sometieron a un prensado manual, con el objetivo de separar la porción líquida de la masa de frutos, y posteriormente se tomó una muestra para cada racimo.
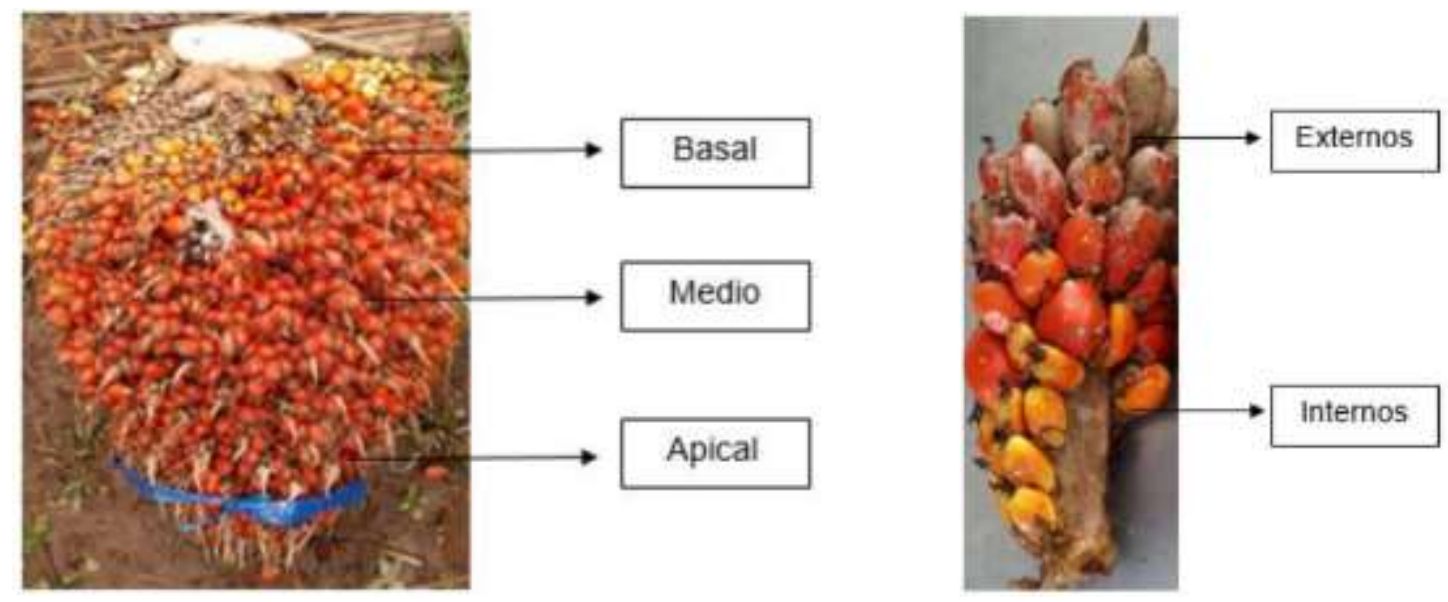

Figura 3. Secciones de muestreo del racimo

Para realizar la caracterización fisicoquímica del aceite crudo de palma aleatoriamente se analizaron cinco muestras del mismo código genético; los ensayos se basaron en los métodos descritos en el manual de laboratorio de planta de beneficio de Cenipalma (Fedepalma, 2006), en los cuales se determinó: Punto de fusión, ácidos grasos libres (AGL), humedad y material volátil, perfil de ácidos grasos, impureza del aceite y potencial de aceite. Se realizó la prueba de impureza en el aceite por medio de un montaje de filtración al vacío con embudo de Buchner y Kitasato, en el cual se retira el material insoluble de una muestra fluida, en este caso aceite de palma, con el fin de determinar el porcentaje de impurezas con la que se está procesando la extracción del aceite crudo de palma. El potencial de aceite se analizó mediante la determinación del porcentaje de 
extracción, con ello se conoce la factibilidad de la extracción, además sirve para comparar el rendimiento de extracción entre el aceite comercial y el aceite hibrido.

Finalmente, los valores registrados fueron comparados con los del aceite de palma africana según la Norma Técnica Colombiana para aceite crudo natural de palma africana NTC 431 (ICNTC, 2009).

\section{RESULTADOS Y DISCUSIÓN}

\section{Caracterización fisicoquímica del aceite crudo de palma}

Las propiedades fisicoquímicas del aceite de palma de frutos provenientes de la empresa Hacienda La Cabaña, del municipio de Cabuyaro del departamento del Meta, se encuentran dentro de lo esperado para este tipo de aceite; los resultados se muestran en la Tabla 1. De acuerdo a los análisis realizados se obtuvo que el punto de fusión está entre 21 y $29^{\circ} \mathrm{C}$; la cantidad de ácidos grasos libres (expresado como ácido palmítico) es $4.23 \%$; la humedad y material volátil $0.25 \%$; y la impureza del aceite en promedio es $0.07 \%$.

Tabla 1. Propiedades fisicoquímicas del aceite crudo de palma código Fortuna

\begin{tabular}{lcc}
\hline \multicolumn{1}{c}{ Parámetro } & Valor & Unidad \\
\hline Punto de fusión & $21-29$ & ${ }^{\circ} \mathrm{C}$ \\
Ácidos grasos libres (AGL) & 4.23 & $\%$ \\
Humedad y material volátil & 0.25 & $\%$ \\
Impureza del aceite & 0.07 & $\%$ \\
Potencial del aceite & 28.80 & $\%$ \\
\hline
\end{tabular}

Los valores del potencial de aceite fueron determinados por el departamento agronómico en el área de investigación de la empresa Hacienda La Cabaña S.A., quienes realizan rutinariamente este análisis a los racimos; de acuerdo con los datos obtenidos para el año 2012, el valor del potencial de aceite registrado fue $28.80 \%$. El alto potencial del racimo depende en gran medida de la cantidad de fruto presente en el racimo, el mayor peso promedio de sus frutos, el alto contenido de pulpa en sus frutos y de aceite en pulpa. 


\section{Perfil de ácidos grasos}

El análisis del perfil de ácidos grasos fue realizado en el Laboratorio de Cenipalma. En la Tabla 2 se muestran los ácidos grasos observados y sus cantidades presentes en el aceite, teniendo en cuenta su clasificación química.

Tabla 2. Ácidos grasos incluidos en el perfil de ácidos grasos y su clasificación

\begin{tabular}{lcc}
\hline \multicolumn{1}{c}{ Ácido graso } & Fórmula & Contenido (\%) \\
\hline Saturados & & \\
Mirístico & $\mathrm{C} 14: 0$ & 0.16 \\
Pentadecanoico & $\mathrm{C} 15: 0$ & 0.04 \\
Palmítico & $\mathrm{C} 16: 0$ & 23.90 \\
Margárico & $\mathrm{C} 17: 0$ & 0.09 \\
Esteárico & $\mathrm{C} 18: 0$ & 2.62 \\
Araquídico & $\mathrm{C} 20: 0$ & 0.33 \\
Monoinsaturados & & \\
Palmitoléico & $\mathrm{C} 16: 1$ & 0.29 \\
Oleico & $\mathrm{C} 18: 1 \mathrm{n} 9 \mathrm{c}$ & 5.00 \\
Polininsaturados & & \\
Linoléico & $\mathrm{C} 18: 2 \mathrm{n} 6 \mathrm{c}$ & 11.92 \\
a-Linoléico & $\mathrm{C} 18: 3 \mathrm{n} 3$ & 0.38 \\
\hline
\end{tabular}

Los resultados obtenidos corresponden específicamente a un periodo de análisis del segundo semestre del año, donde las variaciones encontradas pueden atribuirse a factores como el tipo de cultivo, región agrícola, condiciones climáticas, sistema de producción, procesos y almacenamiento entre otros.

\section{Comparación con las características teóricas del híbrido interespecífico classic}

En la Tabla 3 se observan los valores de algunos parámetros de los aceites de palma de alto oleico y el aceite de palma código Fortuna. El punto de fusión teórico encontrado esta entre 22 y 28 , el de código Fortuna entre 21 y 29 . La 
humedad y el material volátil hallado fueron de $0.25 \%$, teniendo como referencia un valor máximo de $0.5 \%$. En cuanto al porcentaje de impurezas insolubles se halló un valor de $0.07 \%$, siendo el valor máximo teórico de $0.10 \%$.

Tabla 3. Comparación del aceite de palma alto oleico y aceite de palma código Fortuna

\begin{tabular}{lccc}
\hline \multicolumn{1}{c}{ Parámetro } & Unidad & Aceite de palma OxG & Código Fortuna \\
\hline Punto de fusión & ${ }^{\circ} \mathrm{C}$ & $22-28$ & $21-29$ \\
Humedad + Material volátil & $\%$ & 0.5 máx. & 0.25 \\
Impurezas insolubles & $\%$ & 0.10 máx. & 0.07 \\
Ácidos grasos libre (AGL) & $\%$ & 5.0 máx. & 4.23 \\
(como ácido palmítico) & $\%$ & 28.89 & 28.80 \\
Potencial de aceite & $\%$ & $\%$
\end{tabular}

Fuente: Fedepalma, (2013); Zapata, (2010); Duran et al., (2004)

Los factores que tienen mayor incidencia en el deterioro oxidativo del aceite de palma crudo durante su extracción y almacenamiento son: la presencia de trazas de metales como hierro y cobre, el sobrecalentamiento del aceite en presencia de oxígeno, la exposición directa a la luz solar, y un alto contenido de clorofila proveniente de racimos inmaduros, los cuales impactan directamente sobre la calidad del aceite (Delgado, 2004). En este sentido, el porcentaje de AGL en el aceite de palma crudo es un indicativo de las buenas prácticas llevadas a cabo durante los procesos de extracción y almacenamiento, y el transporte desde las plantas de beneficio hasta las plantas refinadoras, así como del estado del fruto procesado (Rincón y Martínez, 2009). En el caso del contenido de AGL del aceite de palma código Fortuna se obtuvo como resultado $4.23 \%$, lo cual es un indicativo de las buenas prácticas llevadas a cabo durante los procesos de extracción, almacenamiento, y transporte, así como del estado del fruto procesado. Las industrias de refinación de aceites y grasas buscan un bajo contenido de ácidos grasos libres (AGL) (Rincón y Martínez, 2009). De acuerdo con los datos obtenidos del potencial de aceite se tiene que el valor teórico de la palma comercial y el registrado para el hibrido Fortuna son muy similares (Tabla 4), 
puesto que ambas lecturas fueron tomadas cuando los racimos estaban en su estado de madurez óptimo.

Tabla 4. Comparación del perfil de ácidos grasos del aceite de palma alto oleico y el aceite de palma código Fortuna

\begin{tabular}{lcc}
\hline \multicolumn{1}{c}{ Ácidos grasos } & $\begin{array}{c}\text { Aceite de palma O x G } \\
(\%)\end{array}$ & $\begin{array}{c}\text { Aceite de palma } \\
\text { Fortuna }(\%)\end{array}$ \\
\hline C12:0 Láurico & 0.39 & 0.00 \\
C14:0 Mirístico & 0.60 & 0.16 \\
C16:0 Palmítico & 28.50 & 23.90 \\
C16:1 Palmitoléico & 0.40 & 0.29 \\
C18:0 Esteárico & 3.50 & 2.62 \\
C18:1 Oleico & 54.20 & 59.00 \\
C18:2 Linoléico & 11.60 & 11.92 \\
C18:3 Linoléico & 0.20 & 0.38 \\
C20:0 Araquídico & 0.30 & 0.33 \\
\hline
\end{tabular}

Fuente: Zapata, (2010).

En comparación con la palma de aceite comercial, el código Fortuna tiene más triglicéridos que contienen ácido oleico (59 Vs 54.2), linoleico C18:2 (11.9 Vs 11.6), linoleico C18:3 (0.38 Vs 0.2); un menor contenido de ácido láurico (0 Vs 0.39), mirístico (0.16 Vs 0.6 ), palmítico ( 23.9 Vs 28.5 ), palmitoléico ( 0.29 Vs 0.4 ) y esteárico (2.62 Vs 3.5); y un valor relativamente similar para el ácido araquídico (0.3 Vs 0.33 ). En general no se evidencia grandes diferencias en el contenido de ácidos grasos, lo cual le confiere ventajas nutricionales, utilizando técnicas similares para la extracción del aceite de palma alto oleico.

En relación con los componentes de ácidos grasos del aceite de palma, el hibrido Fortuna conserva sus valores relativamente muy similares al de la palma comercial. El primero tiene más triglicéridos que contienen ácido oleico (59 Vs 40), y un menor contenido de ácido palmítico (23.9 Vs 44). Estos resultados dan por confirmado las características del aceite de palma alto oleico (Tabla 5). 
Tabla 5. Comparación del perfil de ácidos grasos del aceite de palma y el aceite de palma código Fortuna

\begin{tabular}{lccc}
\hline \multirow{2}{*}{ Ácidos grasos } & \multicolumn{2}{c}{ Aceite de palma NTC 431 } & Código Fortuna \\
\cline { 2 - 4 } & $\mathbf{( \% )}$ & Rango & $(\%)$ \\
\hline C12:0 Láurico & 0.40 & 0.4 & 0.00 \\
C14:0 Mirístico & 1.25 & $0.5-2.0$ & 0.16 \\
C16:0 Palmítico & 44.0 & $41.0-47.0$ & 23.90 \\
C16:1 Palmitoléico & - & $<0.6$ & 0.29 \\
C18:0 Esteárico & 4.75 & $3.5-6.0$ & 2.62 \\
C18:1 Oleico & 40.0 & $36.0-44.0$ & 59.00 \\
C18:2 Linoléico & 10.0 & $8.0-12.0$ & 11.92 \\
C18:3 Linoléico & - & $<0.5$ & 0.38 \\
C20:0 Araquídico & - & $<0.1$ & 0.33 \\
\hline
\end{tabular}

Fuente: ICNTC, (2009)

Los ácidos grasos insaturados que constituyen los triglicéridos (TG) del aceite de palma son el oleico (36-44\%) y el linoleico (9-12\%) que tienen configuración cis; también posee los ácidos grasos saturados palmítico (39.3-47.5\%) y esteárico (3.5-6\%) (Rincón y Martínez, 2009), lo cual coincide con lo observado en el presente estudio para el hibrido Fortuna.

En lo fundamental, la composición de los triglicéridos del aceite es: 1-palmitoil-2,3dioleoil-sn-glicerol (alrededor de 23\%) y 1,3-dipalmitoil-2-oleoilsn-glicerol (cerca del $18 \%$ ). Dichos triglicéridos se caracterizan porque el ácido oleico se encuentra en mayor proporción en la posición sn-2 del TG, lo que proporciona una mejor biodisponibilidad de ácidos grasos monoinsaturados. La importancia de la biodisponibilidad de los ácidos grasos insaturados oleico y linoleico radica en que estos son hipocolesterolemiantes, y por tanto disminuyen las concentraciones de colesterol LDL (fracción perjudicial del colesterol que en exceso se deposita en la paredes arteriales originando la formación de ateromas), y potencian las acciones beneficiosas del colesterol HDL (encargado de recolectar el colesterol libre desde los tejidos del cuerpo hasta el hígado, donde es descompuesto y eliminado del 
cuerpo por medio de la bilis) (Stanley, 2008). Además, como el aceite de palma posee una mínima cantidad de los ácidos grasos láurico $(<5 \%)$ y mirístico $(0.5$ $2 \%$, que también se observó en el presente estudio, y que son considerados hipercolesterolémicos, no genera un aumento del colesterol en la sangre (Firestone, 2006).

Los antioxidantes también son de gran importancia en la estabilidad del aceite, porque inciden en las propiedades del producto desde el momento mismo de la cosecha y el procesamiento, hasta la formulación de los productos finales, puesto que actúan inhibiendo o retardando el proceso de peroxidación lipídica sobre los ácidos grasos insaturados, durante el cual se hidroliza el enlace éster de los triglicéridos y se liberan ácidos grasos que generan peróxidos e hidroperóxidos, principales responsables de cambios en las características organolépticas del producto.

\section{CONCLUSIONES}

Uno de los principales factores que determinan la importancia de los aceites en el componente nutricional de un individuo es el perfil lipídico, el cual está dado por el tipo y proporción de ácidos grasos y componentes minoritarios como pigmentos, vitaminas entre otros; en este sentido el aceite de palma tradicional y el del hibrido Fortuna conservan valores relativos muy similares de ácidos grasos, sin embargo el último tiene más triglicéridos que contienen ácido oleico, y un menor contenido de ácido palmítico, lo cual podría ser favorable desde el punto de vista nutricional

La evidencia de un alto contenido de ácido oleico supone ventajas técnicas y comerciales en la agroindustria, teniendo en cuenta que es un compuesto que juega un papel importante en la salud humana al momento de consumirlo, respondiendo a la tendencia actual de la industria de aceites comestibles de desarrollar productos libres de ácidos grasos trans.

La acidez es uno de los parámetros de calidad más importantes, en el caso del aceite crudo de palma tiene un parámetro límite de $\mathrm{pH}=4$; cuando los resultados 
son mayores se deben buscar las causas, y proveer las acciones correctivas para evitar la rancidez del aceite y daños posteriores.

La humedad es determinante para conocer si el producto se encuentra bajo los estándares de calidad, teniendo en cuenta que este factor afecta la calidad del aceite porque puede crear un medio afable para la proliferación de microorganismos que deterioran el producto.

El porcentaje de ácidos grasos libres (AGL) en el aceite de palma crudo es un indicativo de las buenas prácticas llevadas a cabo durante los procesos de extracción y almacenamiento, y el transporte desde las plantas de beneficio hasta las plantas refinadoras, así como del estado del fruto procesado.

\section{RECOMENDACIONES}

Las evaluaciones realizadas en la composición fisicoquímica del aceite de palma de código Fortuna, indican que hay perspectivas interesantes desde el punto de vista agroindustrial para su utilización en la elaboración de bienes de consumo básico o como materia prima para otras industrias, por lo cual se requieren hacer evaluaciones a escala piloto.

\section{REFERENCIAS BIBLIOGRÁFICAS}

1. Arveláez, A; Mieres, A; Hernández, C. Diseño experimental aplicado a la extracción de aceite de la almendra del corozo (Acrocomia aculeata). Revista Técnica de la Facultad de Ingeniería Universidad de Zulia, 31 (Especial): 122129. 2008.

2. Brugés, C. Estimación y reducción de pérdidas en el proceso de extracción de aceite de palma en la planta de beneficio de Fruto Palmar Santa Elena S.A. (Tumaco). Tesis Ingeniero de producción agroindustrial. Universidad de la Sabana. Facultad de ingeniería. Bogotá, Colombia, 98 p. 2004.

3. Centro Nacional de Investigación en Palma de Aceite (CENIPALMA). Guía sobre el aceite de palma y sus aplicaciones. Bogotá D.C., Colombia. 2013.

4. Centro Nacional de Investigación en Palma de Aceite (CENIPALMA). Guía para el análisis de racimos de palma de aceite. Convenio SENA, SAC FEDEPALMA. 2006.

5. Contreras, Á.; Cayon, G.; Corchuelo, G. Models to estimate the bunch dry weight in African oil palm (Elaeis guineensis Jacq.), American oil palm (Elaeis oleifera H.B.K. Cortes) and the Interspecific Hybrid (E. oleifera $x \mathrm{E}$. guineensis). Agronomía Colombiana, 30 (1): 46-51. 2012. 
6. Cuellar, M. Experiencias en la producción y mercadeo del aceite de palma alto oleico. 28 p. 2015. Recuperado 28 Marzo 2019. Disponible En: http://web.fedepalma.org/sites/default/files/files/3\%20\%20Monica\%20Cuellar.p df

7. Delgado, W. Por qué se enrancian las grasas y los aceites. Revista Palmas, 25 (2): 35-43. 2004.

8. Durán, Q.; Sierra, G.; García, J. Potencial de aceite en racimos de palma de aceite de diferente calidad y su influencia en el potencial y extracción de aceite en la planta de beneficio. Revista Palmas, 25 (II): 501-508. 2004.

9. FEDERACION NACIONAL DE CULTIVADORES DE PALMA DE ACEITE (FEDEPALMA). Acuerdo N. 251 del 2, mayo de 2013. "Por el cual se establecen las características técnicas que deben cumplir los productos objeto de las compensaciones de estabilización". Bogotá, D.C., Colombia. 14 p. 2013.

10. FEDERACIÓN NACIONAL DE CULTIVADORES DE PALMA DE ACEITE. (FEDEPALMA). Planes de Desarrollo Municipal y la agroindustria de la palma de aceite. Bogotá, D.C., Colombia, 56 p. 2016.

11. FEDERACIÓN NACIONAL DE CULTIVADORES DE PALMA DE ACEITE. (FEDEPALMA). Manual de laboratorio plantas de beneficio primario para fruto de palma de aceite. 117 p. 2000.

12. FEDERACIÓN NACIONAL DE CULTIVADORES DE PALMA DE ACEITE. (FEDEPALMA). La palma de aceite en el Departamento del META. 1 p. 2016.

13. FEDERACIÓN NACIONAL DE CULTIVADORES DE PALMA DE ACEITE. (FEDEPALMA). La palma de aceite en Colombia. 1 p. 2016.

14. FEDERACIÓN NACIONAL DE CULTIVADORES DE PALMA DE ACEITE. (FEDEPALMA). Herramienta del Sistema de información estadística del sector palmero (Sispa). Recuperado 28 Marzo 2019. Disponible En: http://web.fedepalma.org/sispa

15. Firestone, D. Physical and chemical characteristics of oils, fats, and waxes, $2^{\text {nd }}$ Ed, AOCS Press (Estados Unidos). 2006.

16. Fontalvo, M.; Vecino, R.; Barrios, A. El aceite de palma africana Elaeis guineensis: Alternativa de recurso energético para la producción de biodiesel en Colombia y su impacto ambiental. Prospectiva, 12 (1): 90-98. 2014.

17. INSTITUTO COLOMBIANO DE NORMAS TÉCNICAS Y CERTIFICACIÓN (ICNTC). NTC 431, Aceite crudo de palma africana (Elaeis guineensis Jacq). Bogotá D.C. 11 p. 2009.

18. Martínez, G. Panorama de la agroindustria palmera, retos y oportunidades. En: Seminario "La agroindustria de la palma de aceite: un negocio sostenible e inclusivo". Bogotá, Colombia 67 p. 2013.

19. Mesa, J. El aceite de palma: la alternativa de los aceites y grasas en América. Revista Palmas, 25 (I): 15-28. 2004.

20. Núñez, D. Uso de residuos agrícolas para la producción de biocombustibles en el departamento del Meta. Tecnura, 16 (34): 142-156. 2012.

21. Ortiz, R.; Fernández, O. El cultivo de la palma aceitera. Editorial Universidad Estatal a Distancia, 1aㅡ. Ed. San José, Costa Rica, 208 p. 1994.

22. Prada, F.; Romero, A. Muestreo y análisis de racimos en el cultivo de la palma de aceite. Centro Nacional de Investigación en palma de aceite (Cenipalma); 
Sistema Nacional de Aprendizaje (SENA); Sociedad de Agricultores de Colombia (SAC), Bogotá. 158 p. 2012.

23. Quesada, G. Tecnología de palma aceitera. Cultivo e industria de la palma aceitera (Elaeis guineensis). s.f. Recuperado 28 Marzo 2019. Disponible En: http://www.mag.go.cr/bibioteca virtual ciencia/tec palma.pdf

24. Rincón, S.; Martínez, D. Análisis de las propiedades del aceite de palma en el desarrollo de su industria. Revista Palmas, 30 (2): 11-24. 2009.

25. Rivera, Y.; Moreno, A.; Romero, H. Biochemical and physiological characterization of oil palm interspecific hybrids (Elaeis oleifera $x$ Elaeis guineensis) Grown in hydroponics. Acta Biológica Colombiana, 18 (3): 465472. 2013.

26. Stanley, J. The nutritional reputation of palm oil. Lipid Technology, 20 (5): 112114. 2008.

27.Zapata, L. Situación y perspectivas del aceite de palma alto oleico OxG en Colombia. Revista Palmas, 31 (II): 349-353. 2010. 